\title{
Design and Development of Solar Powered Quadcopter using 3D Printing Technology
}

\author{
Mr. Hezil Pramod* \\ * (Department of Mechanical Engineering, VTU, Cambridge Institute of technology, Bengaluru
}

\begin{abstract}
This solar powered Quadcopter is aimed to collaborate the concepts of photovoltaics and Quadcopter, thereby creating one device that combines all these technologies, to create a product that has the capabilities of that of a traditional battery powered Quadcopter. The major concepts explored through this project are the functionalities, capabilities and the production of solar cell power of Quadcopter. The usage of 3D printing technology has made it possible to decrease the weight of the Quadcopter without compromising on its strength. The change in the design from $\mathrm{X}$ to $\mathrm{H}$ shape has not only improved the stability but has also decreased the system complexity. With the use of simple mechanisms, the Quadcopter can be made to move on the ground as well. This project has made the device economical and efficient to use, filling the requirement gaps of the traditional Quadcopter.

Keywords: 3D printing technology, solar power, UAV
\end{abstract}

\section{INTRODUCTION}

The Traditional Quadcopters require replacements or removal of batteries followed by their charging, this involves a lot of time and effort. The Quadcopter used here includes solar powered adaptor that charges the drained battery without involving its removal, it is also used to regulate the current given to the battery.

Quadcopter is an Unmanned Aerial Vehicle which consist of a base controller and a common system between the two. The Quadcopter is operated by varying the spin RPM of its 4 rotors to lift and control the torque, this thrust from the device helps to maneuver the device and helps to keep it airborne. The Unmanned Aerial Vehicles are either used under remote control by a human or used intermittently by computers [1].

The different functional categories of Unmanned Aerial Vehicle include Target and decoy, Reconnaissance, Combat, Logistics, Research and Development, Civil and Commercial Unmanned Aerial Vehicles. Unlike manned aircrafts, Unmanned Aerial Vehicles were mostly used for missions that were considered dangerous for humans. Although they are more widely used in military applications, their use is expanding to recreational, commercial, agricultural, scientific and other purposes.

\section{LITERATURE SURVEY}

The first Quadcopter used was a multicopter with 4 rotors and 8 propellers driven by one engine, this showed considerable stability in its time. Later, an X shaped structure was developed with smaller propellers used for thrust and control, but it's maximum height was $5 \mathrm{~m}$. Along came much larger prototype used for civil and military purposes, this was the first Quadcopter to demonstrate a successful forward flight, but it faced lack of orders due to its weight [2].

\section{3D PRINTING TECHNOLOGY}

3D printing is the next generation of printing technologies that can create or recreate a freely standing structure in a single piece. It involves addition of layers of fabrication. This usually consists of two stages, (1) The transfer of data directly from software to the printed structure, (2) Printing the object layer by layer by placing the print head in all the directions repeatedly.

This process is carried out by first making the design in a CAD system and then printing the areas by compiling $2 \mathrm{D}$ slices that represent a 3D object, thereby printing the object layer by layer until it's completion. The next stage is the manufacturing process that is divided in coating and fusing. Through all these steps, the material is placed over a surface and with the help of an energy source, the layers are created. Depending on the kind of technologies used, the material and energy source vary. The different kind of technologies used to create the layers are different with the different stages of development. These are classified on the basis of material accommodation and strength. They can be broadly thrown into the following categories-

- Stereo-lithography (SLA)

- Fused Description Modelling (FDM)

- Laminated Object manufacturing (LOM)

- Selective Laser Sintering (SLS)

- Direct Metal Sintering (DMLS)

- Ink Jet Printing

- Poly-Jet 3D Printing 


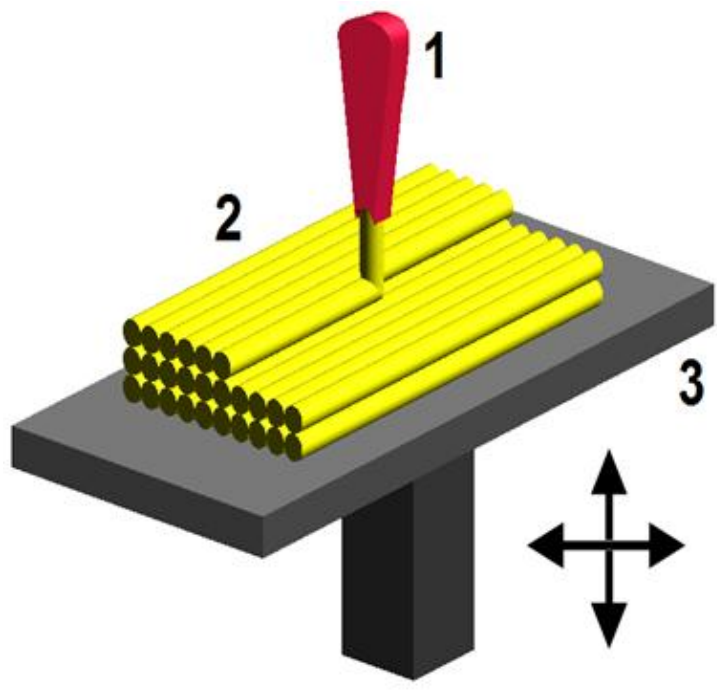

Fig. 1 Fused Description Modelling

1- Nozzle ejecting molten plastic

2- Deposited plastic

3- Controlled and movable table technology digital copy of an object

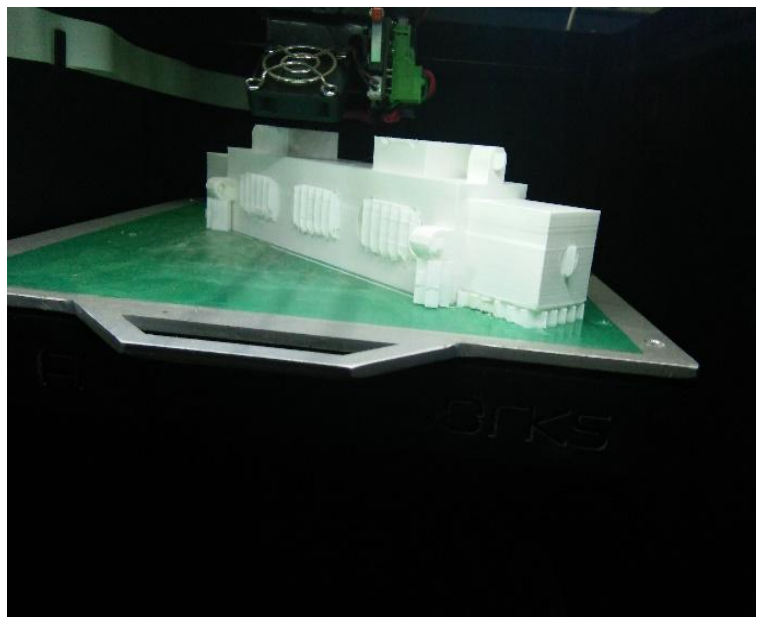

Fig. 2 Body frame printing using 3D printer

In this project, Fusion Description Modelling was used to create the structures. FDM involves the use of plastic or wax that is ejected out through a nozzle that follows the object's crosssectional geometry one layer by another. The nozzle is initially heated so as to melt the plastic and has a mechanism to allow or stop the flow of this melted plastic. It can be made to move in horizontal and vertical paths by mechanisms that are controlled by a CAM (Computer Aided Manufacturing) software. This nozzle is placed on XY plotter mechanism that takes off the contours. Another nozzle is present to provide support for the material. As the nozzle moves over the surface, it deposits a thin layer of extruded plastic. This plastic becomes hard immediately bonds with the other underlying layers.
The object moves vertically downward as the layers are being deposited. The entire temperature is maintained just below that of the melting point of plastic [3].

\section{DESIGN}

The design for the proposed model was made using a software called CATIA. The design can be broadly cast into two parts. One that uses propellers to foster the flight abilities to the Quadcopter and another that uses wheels to enable its motion on the ground.

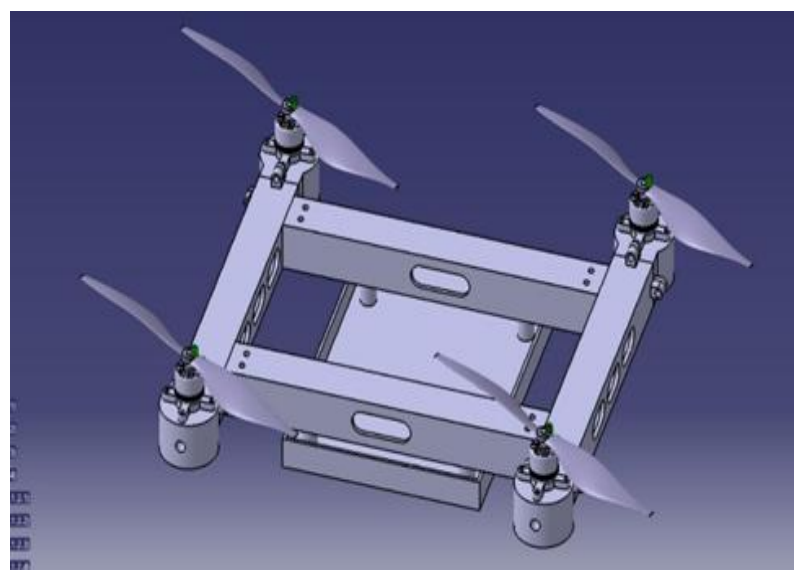

Fig.3 CATIA model with Propellers

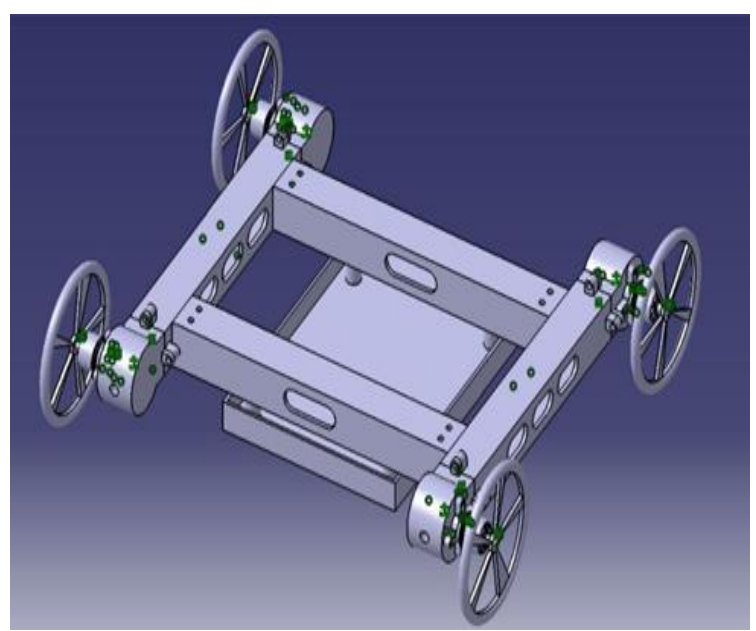

Fig.4 CATIA model with wheels

\section{SPECIFICATIONS}

A description of the specifications of the important parts that are tested and used to build are quadcopter are elaborated in this section. Four motor mounts are used that are $50 \mathrm{~g}$ each, along with 4 motors $920 \mathrm{kv}$ that are $55 \mathrm{~g}$ each. The thrust produced by each of the motors is $920 \mathrm{~g}$. The fibre plate weighs $31 \mathrm{~g}$ with the frame weighing up to $383 \mathrm{~g}$. The design includes four propellers which sums up to $46 \mathrm{~g}$ followed by the four wheels of the Quadcopter which are of $80 \mathrm{~g}$ on the whole. KK 2.1.5 
microcontroller is used whose chip weighs $18 \mathrm{~g}$. Four Electronic Speed Controllers of $30 \mathrm{~A}$ with each weighing $30 \mathrm{~g}$ is used. A solar panel with $12 \mathrm{~V}$ output and $3 \mathrm{~A}$ adapter output weighing $97 \mathrm{~g}$ is used. A $11.1 \mathrm{~V}$ battery source of $2200 \mathrm{mAh}$ power, weighing $146 \mathrm{~g}$ is included with the other parts weighing about $100 \mathrm{~g}$, thus the total weight will be approximately $1400 \mathrm{~g}$. This design has been formulated to considerably reduce the weight of the Quadcopter.

\section{METHODOLOGY}

A thorough research and analysis was done on various designs for the quadcopter before concluding our design for the device. A CAD software tool named CATIA was used to create the quadcopter's design. CATIA was used as it provided as a multi-platform tool to give shape to our design and modify, virtualize, style and create the design by providing a lot of features and ease of usage. The files were converted to STL format using the same software and then the frames were printed using $3 \mathrm{D}$ printing technology.

FDM technology was used to produce the quadcopter's structures. While researching, a lot of comparisons and analysis were done on the kind of plastic to be used. It was then observed PLA plastic was better than aluminum as it is lighter and can be molded to any shape easily. Hence PLA plastic was used to build the frames. The hardware components specified in the design were then assembled on the frame. Assuming the desired weight of the quadcopter, the thrust was calculated. Accordingly, the components to be assembled were decided. $920 \mathrm{kV}$ motors that produce $900 \mathrm{~g}$ of thrust is used. All the other hardware along with the motors were assembled on the Quadcopter and balanced accordingly until it was tested and verified for a smooth flight.

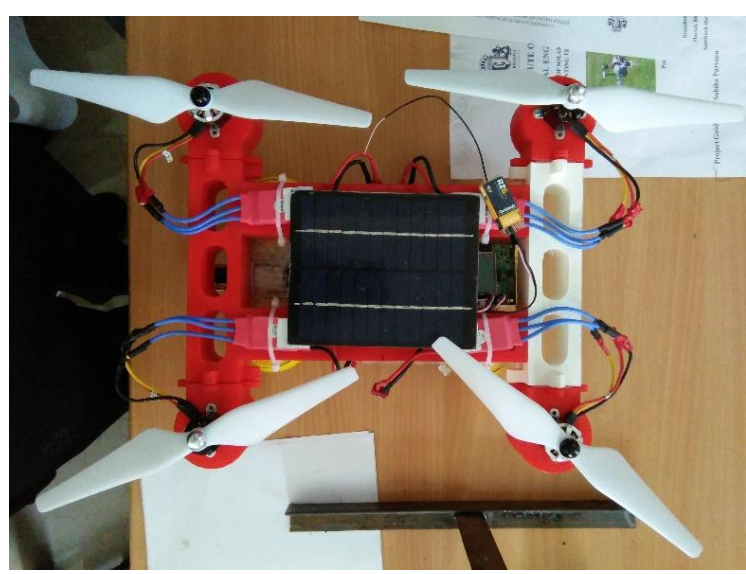

Fig.5 Completed model with Propellers

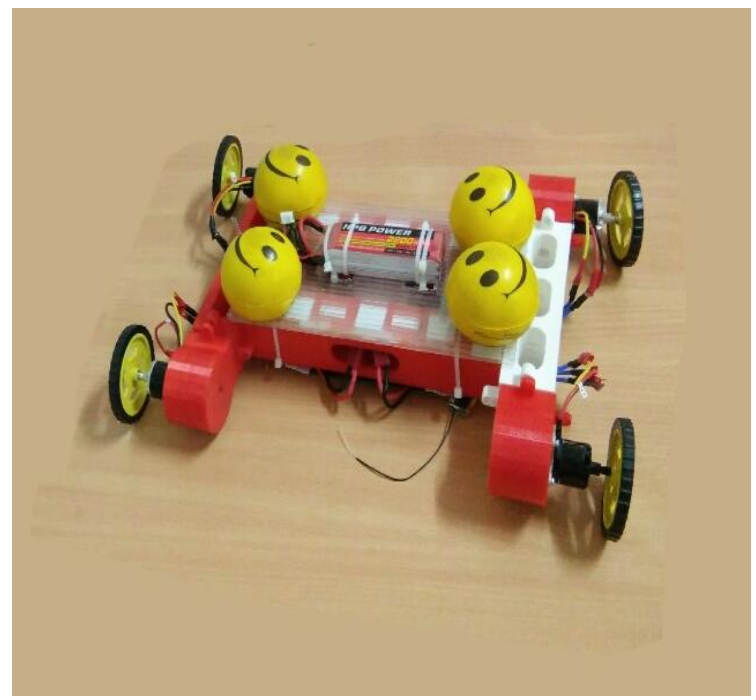

Fig. 6 Completed model with Wheels

VII. APPLICATIONS

The main objectives of this device are energy efficiency, durability and cost efficiency all of which are served without compromising on the strength of the device. Based on this, the Quadcopter can be used in the following fields-

1. Surveillance- It allows people to quickly search and see the areas without having to be there.

2. Search or Rescue Missions- Quadcopters can also serve as spies to track down people's activities and help in rescue missions.

3. Mining- They can be used to regularly inspect power lines, roads and other equipment that provide safety for mining employees. They are also used to detect dangerous pits and entrances to mines where the areas are dangerous and unsafe for mine workers.

4. Product or Food Delivery-This was one of the most recent and eye catching uses of quadcopters that gained a lot of use in restaurants to deliver food to their customers. A lot of companies like Amazon use quadcopters for product delivery to remote places.

5. Research- They are useful for researchers of various universities for verifying and testing new ideas in a number of fields like robotics, flight controls, real time systems, etc.

\section{CONCLUSION}

The 3D printing technology used has made it possible to fabricate a durable and strong structure of decreased weight, compared to most traditional Quadcopters. As this draws its power from a solar source, energy is conserved and also used efficiently, making it cost effective. The modifications in the design that includes change of the Quadcopter's shape from X to H, has resulted in the simplicity of the overall system and has also met the desired stability standards. It has achieved the 
task of linear flight motions successfully and can move effectively on land as well.

\section{FUTURE WORK}

To extend the movement of the Quadcopter in other angles, a steering mechanism can be used. Programming software of the Quadcopter can be further developed to enable the device to make its own decisions such that this can come of use in situations where there is a probability of human errors. A CMOS camera can be used for capturing ground images from bird's eye level and a sensor can be incorporated to foster air surveillance.

\section{REFERENCES}

\section{Journal Papers}

[1] Mohd Khan, "Quadcopter Flight Dynamics" INTERNATIONAL JOURNAL OF SCIENTIFIC \& TECHNOLOGY RESEARCH VOLUME 3, ISSUE 8, AUGUST 2014

[2] . https://en.wikipedia.org/wiki/Quadcopter

[3] Jabbar Qasim Al-Maliki, Alaa Jabbar Qasim Al-Maliki, "The Processes and Technologies of 3D Printing" International Journal of Advances in Computer Science and Technology Volume 4, No. 10, October 2015 